\title{
Necessidades de saúde de familiares de usuários de substâncias psicoativas
}

\author{
Deivson Wendell da Costa Lima ${ }^{1}$, \\ Andreza Carla Queiroz Bezerra Leite², \\ Alcivan Nunes Vieira ${ }^{3}$, Amélia Resende Leite ${ }^{4}$, \\ Margarita Antonia Villar Luis $^{5}$, \\ Lívia Dayane Sousa Azevedo ${ }^{6}$, \\ Juce Ally Lopes de Melo ${ }^{7}$
}

\section{RESUMO}

Buscou-se compreender as necessidades de saúde de familiares de usuários de substâncias psicoativas (SPA). Pesquisa descritiva realizada com familiares dos usuários acompanhados em um Centro de Atenção Psicossocial, cujos dados foram produzidos por meio de entrevistas. Após análise de conteúdo identificaram-se categorias: necessidade de saúde como doença localizada no corpo biológico, necessidade de saúde como reprodução social e necessidade de saúde centrada nas relações e no cuidado. As necessidades de saúde são percebidas como necessidade de cuidado com corpo, mente e espírito. O que não se manifesta por meio de sinais e sintomas nem sempre é reconhecido. Identificou-se tendência em não reconhecer formas de adoecer oriundas do convívio com os usuários de SPA. Há uma demanda de compartilhar problemas com pessoas dispostas a escutar, sejam profissionais ou não. Os profissionais devem estar atentos a estas necessidades que podem implicar no esquecimento dos cuidados com a própria saúde por parte dos familiares.

Descritores: Enfermagem Psiquiátrica; Relações Familiares; Transtornos Relacionados ao Uso de Substâncias; Saúde Mental.

\footnotetext{
${ }^{1}$ Enfermeiro, Mestre em Cuidados Clínicos em Enfermagem e Saúde. Discente do Programa de Pós-Graduação em Enfermagem Psiquiátrica, nível Doutorado, da Escola de Enfermagem de Ribeirão Preto da Universidade de São Paulo. Professor Assistente da Universidade do Estado do Rio Grande do Norte. Mossoró, RN, Brasil. E-mail: deivsonwendell@hotmail.com.

2 Enfermeira. Enfermeira do Hospital Rodolfo Fernandes. Mossoró, RN, Brasil. E-mail: andrezacarla02@outlook.com.

${ }^{3}$ Enfermeiro, Doutor em Cuidados Clínicos em Saúde. Professor Adjunto da Universidade do Estado do Rio Grande do Norte. Mossoró, RN, Brasil. Email: alcivan nunes@yahoo.com.br.

${ }^{4}$ Enfermeira, Mestre em Saúde e Sociedade. Enfermeira da Empresa Brasileira de Serviços Hospitalares. Mossoró, RN, Brasil. E-mail: amelia_resende@hotmail.com.

${ }^{5}$ Enfermeira, Doutora em Enfermagem. Professora Titular da Escola de Enfermagem de Ribeirão Preto da Universidade de São Paulo. Ribeirão Preto, SP, Brasil. E-mail: margarit@eerp.usp.br.

${ }^{6}$ Nutricionista. Mossoró, RN, Brasil. E-mail: liviaazevedo.nutri@gmail.com.

${ }^{7}$ Enfermeira, Mestre em Cuidados Clínicos em Enfermagem e Saúde. Professor Assistente da Universidade do Estado do Rio Grande do Norte. Mossoró, RN, Brasil. E-mail: juceally@hotmail.com.
}

\section{Como citar esse artigo:}

Lima DWC, Leite ACQB, Vieira AN, Leite AR, Luis MAV, Azevedo LDS, et al. Necessidades de saúde de familiares de usuários de substâncias psicoativas. Rev. Eletr. Enf. [Internet]. 2018 [acesso em: ];20:v20a12. Disponível em: https://doi.org/10.5216/ree.v20.47410. 


\section{INTRODUÇÃO}

O uso de Substâncias Psicoativas (SPA) emerge contemporaneamente como uma problemática de abrangência mundial, gerando preocupações no âmbito das políticas públicas de saúde. Neste contexto, a família assume um importante papel para a criação de vínculos, na proteção e socialização da pessoa que faz uso dessas substâncias ${ }^{(1)}$.

As situações enfrentadas pela família que convive com usuários de SPA produzem situações de incompreensão, rejeição, sensação de vulnerabilidade à violência, desentendimentos e desagregação familiar. A convivência com a pessoa que faz uso de SPA pode gerar na família constrangimentos e sentimentos de impotência diante de situações hostis e de tensão, comportamentos violentos e ideias suicidas. A vivência destas situações produz mudanças nos seus hábitos de vida(2).

Isto repercute diretamente no contexto familiar, interferindo não somente nas relações entre a família, mas também nos aspectos emocionais, financeiro, social, estrutural e afetivo ${ }^{(3)}$.

Trata-se de um contexto singular capaz de gerar necessidades de saúde decorrentes do convívio com uma pessoa que faz uso de SPA. As necessidades em saúde estão relacionadas ao adoecimento, sofrimentos individuais, carências ou vulnerabilidades ${ }^{(3)}$.

Necessidades de saúde aqui são entendidas como aquelas direcionadas à preservação da vida das pessoas (como o abrigo, alimentação, convívio social, cooperação entre outras) e aquelas que se voltam para o aprimoramento da condição humana, tais como a liberdade, autonomia, autorrealização dentre outras ${ }^{(4)}$. Contudo todas são histórica e socialmente determinadas, pois o indivíduo nasce e se desenvolve em espaços naturais que envolvem contextos culturais ${ }^{(5)}$.

Essas necessidades de saúde extrapolam categorias identificáveis em nível individual. Portanto, no âmbito coletivo estas necessidades não podem ser isoladas e individualizadas porque assumem um sentido no contexto social onde são geradas. Não se trata de negar a importância da manifestação e satisfação das necessidades individuais específicas, mas sim de reconhecer a demanda por uma ação cuidadora a partir de uma perspectiva em que esses elementos se integram ao fazer profissional ${ }^{(6)}$.

Neste sentido, essa mesma família pode participar ativamente do cuidado com a saúde da pessoa que faz uso de SPA ao apoiar os projetos terapêuticos singulares, de oferecer afeto, segurança e atenção(7). A parceria da família com o serviço de saúde pode ser uma forma de promover o resgate do vínculo familiar desse usuário e estimular a continuidade do seu tratamento(8).

Estas necessidades muitas vezes podem ser negligenciadas quando a atenção está apenas centrada no usuário de SPA. Neste sentido questiona-se: quais são as necessidades de saúde dos familiares das pessoas que fazem uso de SPA? Como estas necessidades emergem a partir das relações familiares estabelecidas no âmbito desta problemática?

Na perspectiva de se fomentar a integralidade em saúde, a identificação das diferentes necessidades de saúde das pessoas, seja em âmbito individual ou coletivo, constitui a estratégia mais adequada para orientar as decisões dos profissionais da saúde e a escolha de suas ações. Além disso, a análise dessas necessidades contribui para a definição de estratégias assistenciais baseadas no contexto e nas relações entre usuários, familiares e comunidade $^{(9)}$. 
A partir destas considerações, este estudo objetivou compreender as necessidades de saúde dos familiares emergidas de sua relação com as pessoas que usam SPA; investigar a percepção sobre necessidade de saúde na visão dessas famílias, e identificar os motivos decorrentes desta relação que os levam a procurar os serviços de saúde.

\section{MÉTODO}

Pesquisa descritiva de abordagem qualitativa realizada em um Centro de Atenção Psicossocial Álcool e Drogas (CAPS ad) de um município do nordeste brasileiro.

A amostra foi composta por 12 familiares de usuários de SPA atendidos no referido serviço, sendo obtida por conveniência ${ }^{(10)}$. Os participantes foram recrutados nas reuniões mensais destinadas aos familiares, onde foi apresentado aos pesquisadores a pesquisa e esclarecidos quanto aos procedimentos da pesquisa.

Os critérios de inclusão foram: familiares de $1 \stackrel{0}{\circ}$ ou de 20 grau, com idade acima de 18 anos e que residissem com o usuário em acompanhamento no CAPS ad. Como critérios de exclusão adotaram-se: familiares de usuários que estivessem em internação hospitalar ou os que faltaram dois encontros consecutivos da coleta de dados.

A quantidade de participantes foi definida também conforme a disponibilidade e a concordância em participar da pesquisa, pois muitos familiares recusaram-se, declarando dificuldades em lidar com a problemática central da pesquisa.

Os dados foram coletados no período de agosto a novembro de 2015, após a assinatura do Termo de Consentimento Livre e Esclarecido, por meio de entrevistas semiestruturadas com as questões norteadoras: 0 que você entende sobre necessidade de saúde? Quais são suas necessidades de saúde? Quais os motivos que te levam a procurar o serviço de saúde?

As entrevistas foram previamente agendadas, realizadas em dois ou três encontros com cada familiar, com média de duração de 45 minutos, em local reservado fora das dependências do serviço de saúde. As falas dos participantes foram gravadas e transcritas na íntegra. Para a análise dos dados empíricos foi utilizada a análise de conteúdo $^{(11)}$ que consiste em um conjunto de técnicas que têm por objetivo analisar a fala utilizando-se de procedimentos (pré-análise, exploração do material e tratamento dos resultados e interpretação) sistemáticos de descrição do seu conteúdo.

Este estudo foi aprovado com parecer 298.697 CAAE no 13975313.2.0000.5294 pelo Comitê de Ética em Pesquisa (CEP) da Universidade do Estado do Rio Grande do Norte (UERN). Para garantir o anonimato dos depoimentos, os participantes foram identificados com a letra "F" de família, seguida de algarismo arábico; esta organização não possui nenhuma referência com a ordem de realização das entrevistas.

\section{RESULTADOS E DISCUSSÕES}

O estudo foi conduzido com participação de dois homens e 10 mulheres cujas idades variaram de 20 a 62 anos, sendo a idade média de 38 anos. Com relação ao grau de parentesco com a pessoa em tratamento no CAPS ad, identificou-se que um era pai, três eram filhos e quatro eram mães; além disso quatro referiram ser esposas, sendo que destas uma relatou ser casada e três afirmaram estar em um relacionamento estável. Quanto a ocupação, três eram donas de casa, três diaristas, dois aposentados, um estudante, um pedreiro e dois 
desempregados. Com relação à escolaridade, seis possuíam ensino fundamental incompleto, quatro sem grau de instrução e dois concluíram o ensino médio.

A análise das entrevistas possibilitou a identificação das seguintes categorias: necessidade de saúde como doença localizada no corpo biológico; necessidade de saúde como reprodução social e necessidade de saúde centrada nas relações e no cuidado.

De modo geral, os resultados obtidos neste estudo levaram à compreensão de necessidades de saúde numa visão restrita às carências, aos problemas de saúde, à ausência de lazer, às condições de vida desfavoráveis, ao desenvolvimento de patologias e àquilo que foi expresso como deficiências dos serviços de saúde.

Percebe-se, portanto, uma aproximação destes resultados com uma perspectiva teórica ${ }^{(9)}$ que articula as necessidades de saúde às condições de vida, ao acesso e consumo de tecnologias, à criação de vínculo afetivo entre usuários e serviço de saúde, e à autonomia dos modos de andar a vida.

Na categoria necessidade de saúde como doença localizada no corpo biológico identificou-se que os familiares associaram a condição de saúde à ausência de patologias localizadas no corpo e na mente, como mostram as falas:

Necessidade de saúde é saúde que a pessoa não tem, ou seja, que precisa de saúde. Por exemplo, eu tenho uma sinusite que incomoda muito, fica doendo me machuca muito, então eu necessito de saúde(F4)

É quando a gente está sentindo alguma coisa, mas agora eu num estou sentindo nada não. Minha mente está bem. Eu acho que num tenho nada não porque eu tomo meus remédios(F8).

Identificou-se que os participantes têm uma compreensão do processo de adoecimento, amparada no discurso biomédico que se faz presente em várias expressões do saber no campo da saúde. Seu pensamento reflete e reforça o entendimento de que a doença se materializa no corpo através de sinais e sintomas circunscritos ao seu funcionamento e a sua estrutura. Tal perspectiva se assemelha à noção de necessidade de saúde enquanto cuidado aos agravos e doença como expressão biológica, excluindo o adoecimento psíquico ou outras formas de adoecer que não se enquadram neste mesmo referencial(12).

Essa abordagem, geralmente aplicada aos termos saúde e doença, se contrapõe ao contexto das famílias, cujo convívio com o usuário de SPA nem sempre harmonioso, é permeado por tensões e conflitos e o meio familiar é o espaço em que as emoções são mais facilmente expressas ${ }^{(13)}$.

Portanto, identificar esta compreensão é fundamental no processo de organização de uma rede assistencial para acolher o usuário de SPA e a sua família, pois, a partir dela podem-se planejar ações de âmbito intersetorial, articuladas ao atendimento individual e familiar. Desta forma, ampliam-se as possibilidades de fortalecer o vínculo entre essa família e o serviço de saúde, na medida em que a assistência ofertada tem como centralidade as suas demandas e necessidades de saúde identificadas no acolhimento dessas pessoas.

$\mathrm{Na}$ fala subsequente a percepção de necessidades se amplia caracterizando a categoria necessidade de saúde como reprodução social:

Minha necessidade é procurar viver bem, ter um lugar para dormir bem, procurar comidas boas, é qualidade de vida mesmo. Então, se a gente está fazendo isso, a gente está prevenindo alguma doença, porque qualquer hora a gente 
pode ficar doente porque me preocupo demais com ele. Eu cuido dele, mas a gente precisa cuidar do nosso corpo, da nossa vida também (F1).

A ideia de necessidade de saúde associa-se aos determinantes sociais e de que forma eles influenciam suas situações de bem estar e de convívio social. Os determinantes sociais são considerados recursos que estabelecem o modo de viver, crescer, adoecer e morrer e ainda são influenciados pelas forças e sistemas que marcam as condições do cotidiano de cada um ${ }^{(14)}$.

A preocupação com o familiar usuário de substâncias psicoativas ou SPA é um sentimento traduzido pelos familiares que atingem várias dimensões de sua vida, como a alimentação, moradia e saúde. Nesse sentido, requer uma compreensão das necessidades dos familiares para estabelecer estratégias que reduzam os eventuais conflitos e as dificuldades existentes na dinâmica familiar ${ }^{(15)}$.

Nesta fala a necessidade ainda é percebida como ausência ou carência de requisitos que propiciam o suporte para a manutenção da saúde.

Necessidade de saúde é quando a gente não tem paz interior, não tem preocupações, quando a gente não estar bem com outras pessoas. Necessidade de saúde eu acho que também envolve a busca de pessoas que me façam bem, que converse quando se tem problemas, que me dê atenção e apoio. Sem essas coisas fica mais difícil de viver bem(F6)

Essa compreensão de necessidade de saúde se expressa em perspectiva ampla, em sua relação com o bemestar como condição para obtenção de saúde, por meio de recursos pessoais, materiais e espirituais ${ }^{(16)}$. A busca pelo afeto e cuidado pelos familiares promove melhoria nas relações familiares e no processo de tratamento do usuário de substâncias psicoativas ou SPA e, consequentemente, na sua qualidade de vida(15).

Houve ainda o relato de que a falta de lazer, de repouso adequado, alimentação e socialização como necessidades de saúde que, quando não atendidas, geram desconforto e dificuldade para seguir os modos de viver. As atividades voltadas para o lazer são consideradas como um importante aspecto para se garantir a saúde.

A necessidade é de uma boa alimentação, é dormir direito, procurar uma coisa que possa divertir um pouco. Para ter saúde é preciso lazer, porque assim é uma vida saudável, para continuar bem, porque senão qualquer um adoece(F2).

Fica evidente na percepção deste familiar que essa necessidade não implica apenas no atendimento das necessidades mais básicas de bem-estar individual e social. Pressupõe o atendimento das condições situadas em um nível que incluem o ser humano em suas várias dimensões e nas relações estabelecidas com a coletividade onde ele está inserido, entendidas como de apoio.

O distanciamento entre o ser individual e social desorganiza a vida em sociedade, uma vez que desconsidera o reconhecimento individual dentro da sociedade. Isso gera o desconforto ou sofrimento para seguir a vida diante de algo que falta ou algo que não vai bem ${ }^{(17)}$.

As modificações no contexto familiar e as dificuldades vividas na reorganização da vida podem ser reflexo da convivência com a pessoa que faz uso de SPA a qual produz necessidades de cuidado que nem sempre são percebidas pelos familiares ou pelos profissionais que os assistem. 
Minha vida é em função dele. Necessidade é fazer minhas coisas, de me cuidar, mas tudo que faço é para ajudar a ele. Faltei meu trabalho agora de manhã para levar ele ao CAPS porque se deixar ele ir só, ele pode ter ataque no meio da rua. Sofro muito com tudo isso e não sei mais o que fazer, também preciso de ajuda(F12).

As necessidades decorrentes do uso de álcool e outras drogas produziram uma relação de codependência entre o usuário de drogas e o membro de sua família. Esse resultado de codependência apresenta similaridade com outros estudos que familiares assumem a responsabilidade de cuidar do usuário de drogas, o que leva a desgastes físicos e sobrecarga emocional, principalmente quando colocam as necessidades do outro acima do próprio $^{(18-19)}$.

O familiar antes de assumir o papel de cuidador, deve encontrar estratégias para enfrentar as situações adversas e buscar conhecer primeiramente seus anseios para depois passar a cuidar do outro. Isso fortalece a relação familiar e influencia no processo terapêutico do usuário de drogas, pois ele vê a família como sua aliada na necessidade de prevenir os riscos advindos do uso das drogas ${ }^{(2,20)}$.

As carências e as dificuldades evidenciadas pelas famílias também estão associadas às relações estabelecidas com a pessoa que faz uso de SPA, as quais podem afetar várias dimensões da vida como a saúde, lazer, trabalho, bem-estar físico e psicológico além do próprio relacionamento entre os familiares. Por esse motivo faz-se necessário o uso de instrumentos de trabalho que permitam a identificação social das necessidades de saúde, tomando estas como centro de suas intervenções e práticas ${ }^{(6)}$.

A categoria necessidade de saúde centrada nas relações e no cuidado surgiu da análise de falas que se remeteram aos vínculos criados entre a pessoa que procura o serviço de saúde e os seus profissionais. Consiste em um campo onde acontece o acesso e consumo de tecnologias leves de saúde consideradas aquelas que instrumentalizam as relações entre profissionais e usuários, o diálogo e a produção dos vínculos ${ }^{(21)}$.

A relação estabelecida entre os usuários e suas famílias com a equipe de profissionais do serviço de saúde insere-se no âmbito das tecnologias leves. Por meio delas podem ser estabelecidos vínculos que tornam a passagem pelos serviços mais acolhedora e resolutiva. O vínculo é estabelecido pela relação de confiança entre os envolvidos, significando o estabelecimento de uma relação contínua em que as subjetividades são compreendidas ${ }^{(22)}$.

A relação familiar com a pessoa que faz uso de SPA é forte devido ao sentimento e dedicação que têm no cuidado com ele:

Por eu cuidar de um dependente, amar muito e preocupar demais com ele, eu tenho que ficar sempre muito atenta. Antes eu não tinha tanto preparo para cuidar dele, hoje eu trabalho na função de ajudar ele, acho que é por isso que nossa relação é forte e eu não tenho coragem de abandonar ele(F7).

Como se eu fosse a mãe dele, porque eu que cuido dele e nós somos muito ligados, tem que ter muito cuidado com ele. Eu sei que ele precisa de mim para se afastar desse mal(F9).

Pode-se observar nas falas a preocupação pelo seu familiar no intuito de garantir seu bem-estar e ajudar no seu tratamento, apesar das dificuldades que esse cuidado parece encerrar e que denotam o grande esforço para assegurar um vínculo e mantê-lo afastado de algo considerado prejudicial à saúde.

As falas denotam a responsabilidade assumida com a pessoa usuária de SPA, a qual gera um sofrimento no âmbito da família. Isto ocorre devido ao laço afetivo existente entre eles porque, em função deste laço, os 
familiares são vistos como corresponsáveis com o dever de ajudar os entes mais próximos. Este achado também foi identificado em outro estudo(3) e reflete um contexto familiar onde o convívio com o usuário de SPA produz outras necessidades de saúde que devem ser acolhidas pelos serviços assistenciais.

O estreitamento de relação entre os membros familiares é um benefício no que se refere ao tratamento da pessoa que faz uso de SPA, porém a responsabilidade assumida pelo ente familiar também pode gerar graves problemas de saúde em quem assume esse cuidado. A procura ou não do serviço de saúde é uma escolha individual e, muitas vezes, as necessidades de saúde não se convertem em demandas para os familiares, porém nem todas as demandas que têm são atendidas pelos serviços, pois estes dificultam o acesso e promovem o distanciamento entre usuários e serviço(9).

Por outro lado, familiares das pessoas que fazem uso de SPA, por vivenciarem sentimentos de incertezas diante das situações de tensão, podem tender a ficar na defensiva e ter dificuldade de procurar o serviço de saúde, mantendo distanciamento e falta de relacionamento com a equipe de saúde ${ }^{(2)}$.

Buscar os serviços de saúde para satisfazer o bem-estar das atividades básicas de vida minimiza o sofrimento, auxilia na resolução de conflitos e proporciona ganho de autonomia para a condução da vida de modo mais confiante, com um maior vínculo com os profissionais da saúde ${ }^{(23)}$. Consequentemente, este familiar tem a necessidade de ser escutado pelos profissionais de saúde para cuidar de seu familiar e de obter acesso a algum tipo de orientações ${ }^{(24)}$.

A minha necessidade é falar, conversar mais com alguém. Já mandaram eu procurar um psiquiatra, mas nunca fui porque ele pode pensar que sou louca, mandar eu fazer exames e me dar remédio controlado(F3).

Nesta fala o participante denota que o sofrimento que poderia ser minimizado com uma escuta acaba sendo intensificado por não se considerar o potencial de cuidado dessa tecnologia e busca-se encaminhar a pessoa para um especialista, o que remete à medicalização numa primeira instância.

A convivência dos familiares participantes com a pessoa que faz uso de SPA gerou algumas necessidades de saúde que culminaram na procura de profissionais da saúde, tanto para cuidar das atividades básicas de vida, como para prevenir problemas de saúde provenientes da relação familiar.

Procurei o serviço quando eu precisava conversar, pedir conselho. Agora eu estou sem apoio. Assim, eu sei preciso de ajuda porque quando a pessoa cuida de outra doente fica doente também. Ai pronto, eu só tenho esse apoio do pessoal do CAPS que eu tenho contato, peço apoio, orientação, converso, pergunto como faço para dormir e comer melhor. Porque é uma equipe muito boa(F5).

Percebe-se que para este familiar a equipe de saúde tornou-se um ponto a mais na sua rede de apoio. Acolher e estabelecer uma relação de vínculo com usuários de SPA e seus familiares tornou-se um desafio que exige respostas dos serviços de saúde, sob a forma de intervenções técnicas e nelas incluída a escuta terapêutica.

Nos serviços de saúde há uma demanda por essa prática que pode ser compreendida como um diálogo, uma conversa, uma interação entre o profissional e o paciente que porta algum tipo de sofrimento. Enquanto uma ferramenta terapêutica a escuta pode atender necessidades de saúde de alguém que apresenta seus medos, ansiedades e tensões ${ }^{(25)}$. 
Ao assumir a condição de protagonista no processo de cuidado da pessoa que faz uso de SPA, a família necessita da parceria de profissionais da saúde atentos às suas necessidades de saúde, além de ofertar condições para que o sofrimento vivido nas relações familiares não seja intensificado ${ }^{(24)}$.

A relação estabelecida entre ações de cuidado dos profissionais e a satisfação das necessidades desses familiares exige a percepção concreta ao modo como estas são traduzidas, apresentadas e incorporadas no trabalho em saúde.

\section{CONSIDERAÇÕES FINAIS}

As necessidades de saúde são percebidas pelos familiares de usuários de SPA como necessidade de cuidado com o corpo, embora também tenham revelado sua preocupação com aspectos que dizem respeito à mente e ao espírito. Aquilo que não se manifesta por meio de sinais e sintomas nem sempre é reconhecido como uma possível necessidade.

Os resultados do estudo também mostraram uma tendência dos participantes em não reconhecer as formas de adoecer oriundas do convívio com os usuários de SPA. Há uma demanda de compartilhar problemas com pessoas dispostas a escutar, sejam profissionais ou não.

Percebeu-se que na sua relação com os usuários de SPA, os familiares experimentam em alguns momentos tensões que geram sobrecarga física e mental. Estabelecer uma relação harmônica entre eles é uma meta a ser incorporada ao acompanhamento desenvolvido pela equipe do CAPS ad. Os profissionais devem estar atentos a estas necessidades que podem implicar no esquecimento dos cuidados com a própria saúde por parte dos familiares.

Identificou-se como temática inovadora a discussão acerca das necessidades de saúde dos familiares dos usuários de SPA, pois, em geral, são negligenciadas por essas pessoas e pelos profissionais de saúde. Essa discussão consiste em uma potencialidade para adoção de estratégias no âmbito dos serviços de saúde no sentido de fortalecer os vínculos entre as famílias e os usuários de SPA com os profissionais. Neste sentido, propõe-se o desenvolvimento de estudos que subsidiem a identificação e a análise dessas necessidades por referenciais teóricos e técnicos.

Uma limitação do estudo pode se referir ao fato de que os participantes serem apenas aqueles que voluntariamente se disponibilizaram a participar. Os outros que eventualmente não estavam satisfeitos com o cuidado recebido pelo CAPS ad, não tiveram oportunidade de se manifestar, pois não se habilitaram a participar da pesquisa.

\section{REFERÊNCIAS}

1. Pandini A, D’artibale EF, Paiano M, Marcon SS. Rede de apoio social e família: convivendo com um familiar usuário de drogas. Ciênc. cuid. saúde [Internet]. 2016 [acesso em: 15 ago. 2018];15(4):716-22. Disponível em:

https://doi.org/10.4025/cienccuidsaude.v15i4.34602.

2. Soccol KLS, Terra MG, Ribeiro DB, Teixeira JKS, Siqueira DF, Mostardeiro SCTS. O cotidiano das relações familiares com indivíduo dependente químico. Cogitare Enferm. [Internet]. 2014 [acesso em: 15 ago. 2018];19(1):116-22. Disponível em:

https://doi.org/10.5380/ce.v19i1.35967.

3. Trindade V, Bartilotti C. "Não quebrou a corrente, mas abriu um elo entre nós": o impacto da dependência química materna sobre o vínculo mãe-filho. SMAD - Revista Eletrônica Saúde Mental Álcool e Drogas [Internet]. 2017 [acesso em: 15 ago.

2018];13(1):4-12. Disponível em: https://doi.org/10.11606/issn.1806-6976.v13i1p4-12. 
4. Mandu ENT, Almeida MCP. Necessidades em saúde: questões importantes para o trabalho da enfermagem. Rev. bras. enferm. [Internet]. 1999 [acesso em: 15 ago. 2018];52(1):54-66. Disponível em: https://doi.org/10.1590/S0034-71671999000100007. 5. Hino P, Ciosak SI, Fonseca RMGS, Egry EY. Necessidades em saúde e atenção básica: validação de instrumentos de captação. Rev. esc. enferm. USP [Internet]. 2009 [acesso em: 15 ago. 2018];43(spe 2):1156-67. Disponível em: https://doi.org/10.1590/S0080$\underline{62342009000600003 .}$.

6. Campos CMS, Bataiero MO. Necessidades de saúde: uma análise da produção científica brasileira de 1990 a 2004 . Interface (Botucatu) [Internet]. 2007 [acesso em: 15 ago. 2018];11(23):605-18. Disponível em: https://doi.org/10.1590/S141432832007000300014.

7. Horta ALM, Daspett C, Egito JHT, Macedo RMS. Vivência e estratégias de enfrentamento de familiares de dependentes. Rev. bras. enferm. [Internet]. 2016 [acesso em: 15 ago. 2018];69(6):1024-30. Disponível em: https://doi.org/10.1590/0034-7167-2015$\underline{0044}$.

8. Alvarez SQ, Gomes GC, Oliveira AMN, Xavier DM. Grupo de apoio/suporte como estratégia de cuidado: importância para familiares de usuários de drogas. Rev. Gaúcha Enferm. [Internet]. 2012 [acesso em: 15 ago. 2018];33(2):102-8. Disponível em: https://doi.org/10.1590/S1983-14472012000200015.

9. Cecílio LCO. As necessidades de saúde como conceito estruturante na luta pela integralidade e equidade na atenção à saúde. In: Pinheiro R, Mattos RA. Os sentimentos da integralidade na atenção e no cuidado à saúde. Rio de Janeiro: IMS-UERJ-ABRASCO; 2001. p. 113-26.

10. Farrokhi F, Mahmoudi-Hamidabad A. Rethinking Convenience Sampling: Defining Quality Criteria. Theory and Practice in Language Studies [Internet]. 2012 [acesso em: 15 ago. 2018];2(4):784-92. Disponível em: https://doi.org/10.4304/tpls.2.4.784-792. 11. Bardin L. Análise de conteúdo. 4a ed. Lisboa: Edições 70; 2009.

12. Deacon BJ. The biomedical model of mental disorder: a critical analysis of its validity, utility, and effects on psychotherapy research. Clin Psychol Rev [Internet]. 2013 [acesso em: 15 ago. 2018];33(7):846-61. Disponível em:

https://doi.org/10.1016/j.cpr.2012.09.007.

13. Paz FM, Colossi PM. Aspectos da dinâmica da família com dependência química. Estud. psicol. (Natal) [Internet]. 2013 [acesso em: 15 ago. 2018];18(4):551-8. Disponível em: https://doi.org/10.1590/S1413-294X2013000400002.

14. Hosseini Shokouh SM, Arab M, Emamgholipour S, Rashidian A, Montazeri A, Zaboli R. Conceptual Models of Social Determinants of Health: A Narrative Review. Iran J Public Health [Internet]. 2017 [acesso em: 15 ago. 2018];46(4):435-446. Disponível em: https://www.ncbi.nlm.nih.gov/pmc/articles/PMC5439032/.

15. Belotti M, Fraga HL, Belotti L. Família e atenção psicossocial: o cuidado à pessoa que faz uso abusivo de álcool e outras drogas. Cad. Ter. Ocup. UFSCar (Impr.) [Internet]. 2017 [acesso em: 15 ago. 2018];25(3):617-25. Disponível em: https://doi.org/10.4322/2526-8910.ctoAR0988.

16. Braun LM, Dellazzana-Zanon LL, Halper SC. A família do usuário de drogas no CAPS: um relato de experiência. Rev. SPAGESP [Internet]. 2014 [acesso em: 15 ago. 2018];15(2):122-44. Disponível em:

http://pepsic.bvsalud.org/scielo.php?script=sci_arttext\&pid=S1677-29702014000200010.

17. Schraiber LB. Necessidades de saúde, políticas públicas e gênero: a perspectiva das práticas profissionais. Ciênc. saúde coletiva [Internet]. 2012 [acesso em: 15 ago. 2018];17(10):2635-44. Disponível em: https://doi.org/10.1590/S1413-81232012001000013. 18. Bortolon CB, Signor L, Moreira TC, Figueiró LR, Benchaya MC, Machado CA, et al . Family functioning and health issues associated with codependency in families of drug users. Ciênc. saúde coletiva [Internet]. 2016 [acesso em: 15 ago. 2018];21(1):1017. Disponível em: https://doi.org/10.1590/1413-81232015211.20662014.

19. Costa B, Marcon SS, Paiano M, Sales CA, Maftum MA, Waidman MAP. Feelings and codependent behavior in the family of illicit drugs users. Acta Scientiarum. Health Sciences [Internet]. 2017 [acesso em: 15 ago. 2018];39(2):175-81. Disponível em:

http://dx.doi.org/10.4025/actascihealthsci.v39i2.27781.

20. Borba LO, Paes MR, Guimarães NA, Labronici LM, Maftum MA. A família e o portador de transtorno mental: dinâmica e sua relação familiar. Rev. esc. enferm. USP [Internet]. 2011 [acesso em: 15 ago. 2018];45(2):442-9. Disponível em: https://doi.org/10.1590/S0080-62342011000200020.

21. Merhy EE, Franco TB. Por uma Composição Técnica do Trabalho em Saúde Centrada no Campo Relacional e nas Tecnologias Leves. Saúde Debate [Internet]. 2003 [acesso em: 15 ago. 2018];27(65):316-23. Disponível em:

https://www.arca.fiocruz.br/bitstream/icict/712/3/Travassos_Viacava_Landmann_Alocacao\%20equitativa_2003.pdf\#page=141.

22. Merhy EE, Chakkour M, Stéfano ME, Santos CM, Rodrigues RA, Oliveira PCP. Em busca de ferramentas analisadoras das tecnologias em saúde: a informação e o dia a dia de um serviço, interrogando e gerindo trabalho em saúde. In: Merhy EE, Onocko R, organizadores. Agir em saúde: um desafio para o público. 3ạ ed. São Paulo: Hucitec; 2007. p. 113-50.

23. Medeiros KT, Maciel SC, Sousa PF, Tenório-Souza FM, Dias CCV. Representações sociais do uso e abuso de drogas entre familiares de usuários. Psicol. estud. [Internet]. 2013 [acesso em: 15 ago. 2018];18(2):269-79. Disponível em:

https://doi.org/10.1590/S1413-73722013000200008.

24. Lisbôa G, Brêda M, Albuquerque M. Ruídos do processo de trabalho e o acolhimento da família na atenção psicossocial em álcool e outras drogas. SMAD - Revista Eletrônica Saúde Mental Álcool e Drogas [Internet]. 2016 [acesso em: 15 ago.

2018];12(2):75-83. Disponível em: https://doi.org/10.11606/issn.1806-6976.v12i2p75-83. 
Lima DWC, Leite ACQB, Vieira AN, Leite AR, Luis MAV, Azevedo LDS, et al.

25. Lima DWC, Vieira AN, Silveira LC. Therapeutic listening in clinical mental health care nursing. Texto contexto - enferm. [Internet]. 2015 [acesso em: 15 ago. 2018];24(1):154-60. Disponível em: https://doi.org/10.1590/0104-07072015002450013. 